\title{
Kikuchi's lymphadenitis and carcinoma of the stomach
}

\author{
J M Radhi, L Skinnider, A McFadden
}

\begin{abstract}
A 37 year old male with a poorly differentiated carcinoma of the stomach presented with cervical lymphadenopathy. It was clinically thought to be a metastatic carcinoma, but on biopsy, it exhibited features of Kikuchi's lymphadenitis. Distinguishing the signet ring histiocytes seen in Kikuchi's disease from signet ring carcinoma cells can be a diagnostic problem. To our knowledge this is the first reported case of an association of Kikuchi's disease and carcinoma.

(F Clin Pathol 1997;50:530-531)
\end{abstract}

Keywords: Kikuchi's disease; cancer; stomach; signet

Department of Pathology, College of Medicine, University of Saskatchewan,

Saskatoon,

Saskatchewan, Canada

J M Radhi

L Skinnider

\section{Department of}

Surgery

A McFadden

Correspondence to: Dr J M Radhi, Department of Pathology, College of Medicine, University of Saskatchewan, Royal

University Hospital, 103

Hospital Drive, Saskatoon Saskatchewan S7N OW8,

Canada.

Accepted for publication 12 March 1997

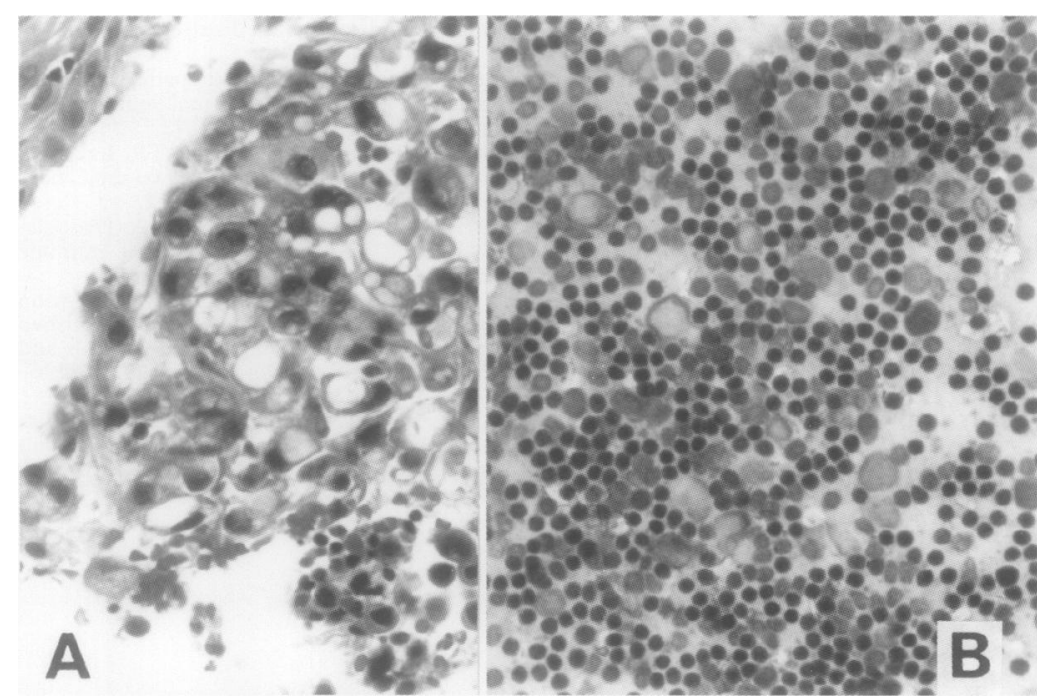

Figure 1 (A) Gastro-oesophageal biopsy showing poorly differentiated carcinoma with signet ring cells. (B) Fine needle aspirate revealed atypical cells with signet ring appearance. vical lymph nodes of young individuals that has a self limited clinical course. ${ }^{12}$ The disease is of unknown aetiology. Suggested causes have been Yersinia entrocolitica, toxoplasma, viral infection, systemic lupus erythematosus, and self limited autoimmune disorders. ${ }^{34}$ Association with ruptured silicone breast implant and malignant fibrous histiocytoma have been described recently. ${ }^{5}$ As the morphological features of Kikuchi's disease are sometimes confused with malignancy, it is important that pathologists are aware of this association.

\section{Case report}

A 37 year old white male presented with a one month history of epigastric pain. The patient was not anorexic but he reported a $7 \mathrm{~kg}$ weight loss. An upper gastrointestinal series was reported as normal. At gastroscopy an ulcer was found on the proximal lesser curvature but biopsy did not show any malignancy. Because of a persistence of symptoms gastroscopy was repeated and this time biopsy showed poorly differentiated adenocarcinoma. Before surgery the patient was noted to have cervical lymphadenopathy. Fine needle aspirate showed atypical cells but was inconclusive for malignancy. A biopsy of one of the cervical lymph nodes showed unusual features consistent with a diagnosis of necrotising lymphadenitis. Preoperative computed tomography confirmed a large lesion of the upper stomach and fairly extensive coeliac axis lymphadenopathy. No hepatic metastases were seen. Oesophagogastrectomy was performed but the patient died following extensive retroperitoneal and mediastinal spread.

\section{Pathological findings}

The initial gastric biopsy showed a poorly differentiated adenocarcinoma with signet ring appearance (fig 1A). Fine needle aspirate of the cervical lymph node revealed lymphoid cells admixed with large atypical cells, some exhibited signet ring appearance. These cells were large round or oval with a crescent shaped nucleus and voluminous pale cytoplasm. Though these cells mimicked the signet ring cell seen in the initial gastric biopsy, the lack of mucin made the diagnosis of metastatic carcinoma very unlikely. The lymph node biopsy revealed paracortical hyperplasia with pronounced mottling appearance and zonal necrosis. The paracortical foci of necrosis were surrounded by aggregates of histiocytes, immunoblasts, lymphocytes, and plasmacytoid monocytes. The necrotic foci contain karyorrhectic debris and lacked neutrophilic infiltrate. The histiocytes observed exhibited crescent and signet ring appearance (fig 2). No haemotoxylin bodies were present. Stains for acid fast bacilli and fungi were negative. The atypical and signet ring cells present in the cervical lymph nodes were negative for cytokeratin, carcinoembryonic antigen, common leucocyte antigen, and $\mathrm{S} 100$ protein; however, these cells were positive for the histiocytic marker CD68 (Dako, Mississauga, Ontario, Canada) (fig 2 , inset). Paracortical area cells expressed T cell markers and residual cortical follicle cells expressed B cell markers. The gastric specimen showed a poorly differentiated adenocarcinoma with involvement of the gastric and omental lymph nodes. 


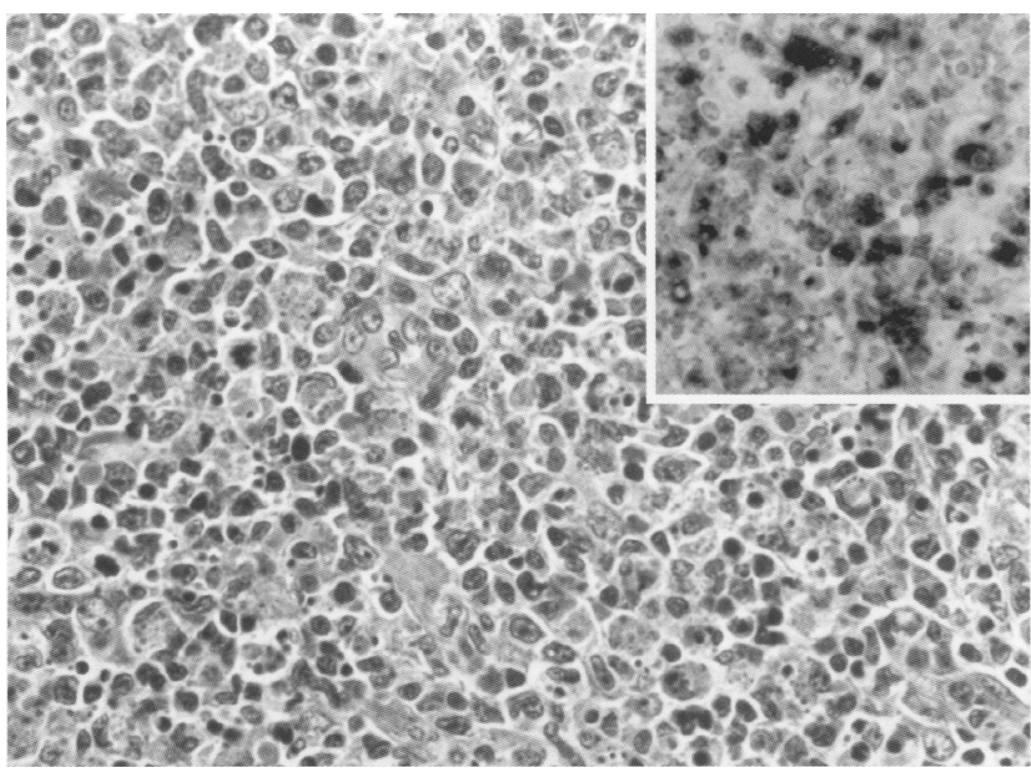

Figure 2 Karyorrhectic debris: karyorrhexis, crescent shaped histiocytes, and immunoblasts. Notice signet ring histiocytes. (Inset) Histiocytes immunostained by antibody CD68 showing strong cytoplasmic staining.

\section{Discussion}

Gastric carcinoma is considered a disease of middle aged or old individuals, but occasionally it affects young people. ${ }^{6}$ Unfortunately the correct diagnosis may be missed even with radiological examination or endoscopy without biopsy because of an unwillingness to consider gastric cancer in young individuals. ${ }^{67}$ Following the establishment of gastric carcinoma, signet ring-type, the present patient was found to have cervical lymphadenopathy. Pathological examination confirmed a necrotising lymphadenitis. The presence of signet ring cells created a diagnostic problem and raised the possibility of metastatic gastric cancer. The nature of these cells became apparent on immunohistochemical staining as these cells expressed CD68, macrophage marker, and were negative for any epithelial markers. The overall appearances were more in keeping with Kikuchi's disease. Necrotising lymphadenopathy was first described in the Japanese literature in 1972 as a benign disorder involving mainly the cervical lymph node. It can be easily mistaken for a malignant process. ${ }^{45}$ Kikuchi's disease is characterised by florid proliferation of activated $T$ cells and histiocytes that can supposedly be produced by diverse diseases including infection, tumours, and various anti- genic stimuli. ${ }^{8}$ A recent report suggests that apoptosis is the mechanism of cell death in Kikuchi's disease and is induced by cytotoxic $T$ cells. ${ }^{9}$ Because it is rather uncommon pathologists have limited experience with fine needle aspiration cytology findings in Kikuchi's disease. In addition none of the features are pathognomonic of Kikuchi's lymphadenitis as systemic lupus erythematosus is included in the differential diagnosis. In Kikuchi's disease the aspirate reveals necrotic debris, phagocytic and non-phagocytic histiocytes, plasmacytoid monocytes, and activated lymphoid cells. ${ }^{10}$ Lymph node biopsy shows paracortical hyperplasia, pale karyorrhectic nodules, with or without extensive coagulative necrosis, and infiltrate similar to those described in fine needle aspirate cytology. Signet ring histiocytes with clear or homogenous lightly amphophilic cytoplasm and compressed nuclei have also been described. ${ }^{1}$ This could mimic signet ring cell adenocarcinoma as encountered in the present case. This case represents the first report of such an association between Kikuchi's disease and diffuse gastric carcinoma. As both share the presence of signet ring cells the morphological differentiation poses a considerable diagnostic challenge.

1 Tsang WYW, Chan JKC, Ng CS. Kikochi's lymphadenitis: a morphologic analysis of 75 cases with special reference to unusual features. Am f Surg Pathol 1994;18:219-31.

2 Jeffe ES. Surgical pathology of the lymph nodes and related organs. Vol 16. In: Jeffe ES, ed. The series major problems in pathology. 2nd ed. Philadelphia: WB Saunders Company, pathology. 2nd

3 Chamulak GA, Brynes RK, Nathwani BN. KikochiFaijimoto disease mimicking malignant lymphoma. $A m \mathcal{f}$ Surg Pathol 1990;14:514-23.

4 Kuo T, Jung SM, Wu WJ. Kikuchi disease of intraparotid lymph nodes presenting as a parotid gland tumor with extranodal involvement of salivary gland. Histopathology 1996;28:185-8

5 Sever CE, Leith CP, Appenzeller J, Foucar K. Kikochi's histiocytic necrotizing lymphadenitis associated with ruptured silicone breast implant. Arch Pathol Lab Med 1996;120. $380-5$.

6 Radi MJ, Fenoglio-Presier CM, Bartow SA, Key CR, Pathak $\mathrm{DR}$. Gastric carcinoma in the young: a clinicopathological and immunohistochemical study. Am $₹$ Gastroentrol 1986; and immur

7 Levine M, Lauler I, Thompson J. Carcinoma of the gastric candida in young people. Am $\mathcal{F}$ Radiol 1983;140:69-72.

8 Chan JKC, Ng CS Kikuchi's histiocytic necrotizing lymphadenitis in the regional lymph nodes of malignant fibrosis histiocytoma: causal or coincidental. Histopathology 1988;123:448-51

9 Tsang WYW, Chan JKC. Fine-needle aspiration cytologic diagnosis of Kikuchi's lymphadenitis. A report of 27 cases Am 7 Clin Pathol 1994;102:454-8.

10 Takakuwa T, Ohnuma S, Koike J, Hoshikawa M, Kuizumi $H$. Involvement of cell-mediated killing in apoptosis in histiocytic necrotizing lymphadenitis (Kikuchi-Fujimoto distiocytic necrotizing lymphadenitis
ease). Histopathology 1996;28:41-8. 